\title{
Guanidine Hydrochloride
}

National Cancer Institute

\section{Source}

National Cancer Institute. Guanidine Hydrochloride. NCI Thesaurus. Code C47551.

The hydrochloride salt form of guanidine, a strong basic compound with parasympathomimetic activity. Guanidine hydrochloride enhances the release of acetylcholine following a nerve impulse and potentiates acetylcholine actions on muscarinic and nicotinic receptors. It also appears to slow the rates of depolarization and repolarization of muscle cell membranes. ( $\mathrm{NCl05)}$ 